\title{
Engel: Internationale Arbeit sehr wichtig
}

\author{
Unter der Präsidentschaft von Anna Lella aus Polen trafen sich Delegierte aus 27 Ländern der \\ ERO-Zone (ERO = European Regional Organisation der FDI World Dental Federation) zu ihrer \\ diesjährigen Vollversammlung in Genf.
}

In der deutschen Delegation unter Dr. Peter Engel vertraten den Freien Verband Deutscher Zanärzte Dr. E.-J. Otterbach, Vorsitzender der Arbeitsgruppe „Freie zahnärztliche Berufsausübung“ und Dr. Thomas Wolf, Mitglied des FVDZ-Bundesvorstandes.

Otterbach und Wolf stellten erste Ergebnisse einer Umfrage der Arbeitsgruppe unter dem Thema „Liberal independent practice ain times of modern forms of dental occupation within the ERO zone of the FDI World Dental Federation" vor.

Mit der Umfrage sollen Antworten auf ERO-Ebene gefunden werden zu Fragen der Versorgungsdichte, Größe von Praxen, Feminisierung des Berufsstandes, Versorgungszentren, Organisationsstrukturen der Zahnärzte oder Universitäten.

Basierend auf diesen Ergebnissen soll eruiert werden, inwieweit Veränderungen in den Berufsausübungsformen und dem
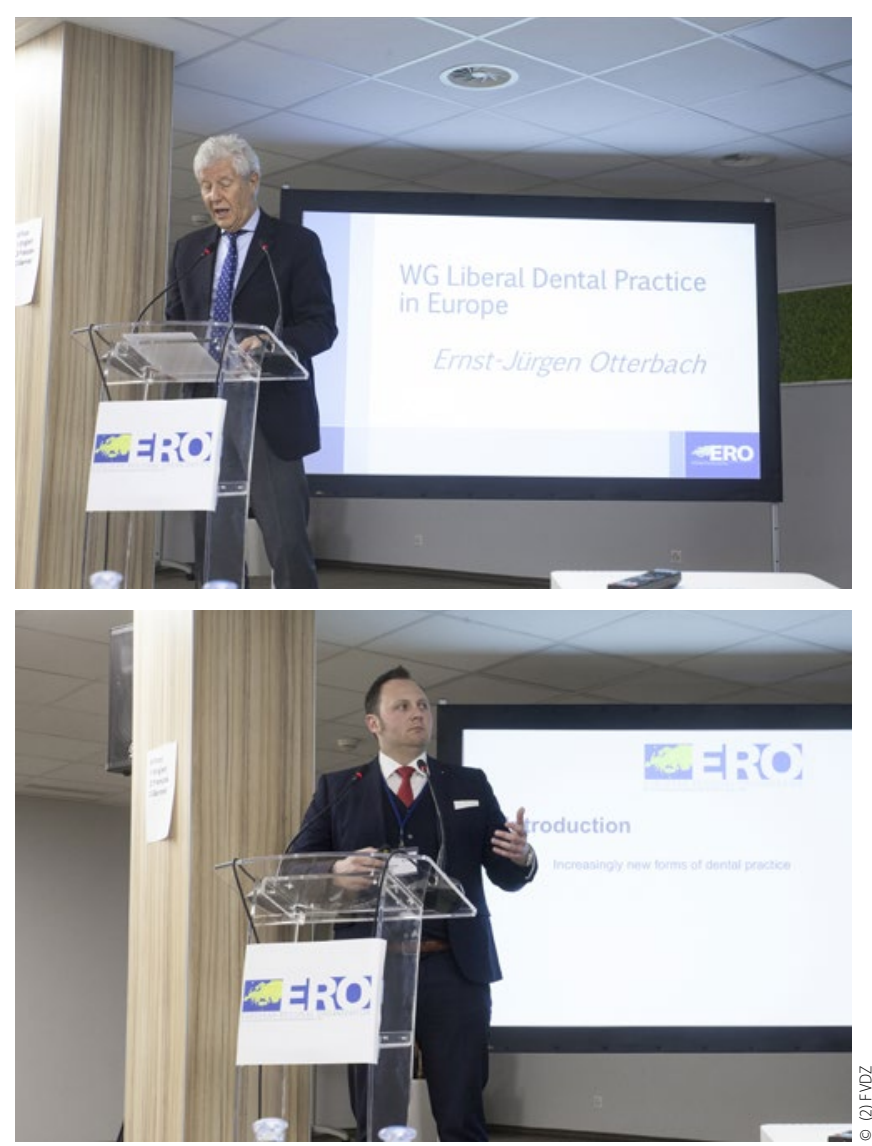

oben: Dr. E.-J. Otterbach, Vorsitzender der Arbeitsgruppe „Freie zahnärztliche Berufsausübung", unten: Dr. Thomas Wolf, Mitglied des FVDZ-Bundesvorstands bei der ERO-Plenarsitzung
Berufsbild eintreten und inwieweit diese Veränderungen eine freie Berufsausübung gefährden.

Ein weiteres zentrales Thema war ein Statement der Arbeitsgruppe „Dental Team“ zum Berufsbild des Zahntechnikers und seiner Beziehung zum Zahnarzt. Das Dental Team definierte das Berufsbild darin neu, mit einer eindeutigen Absage an jegliche Art von Diagnose, Planung oder Behandlung der Patienten durch den Zahntechniker.

\section{Behandlungsbedarf Älterer nimmt stark zu}

In der neuen Arbeitsgruppe „Ageing Population“ wurde der stark zunehmende Behandlungsbedarf der älteren Generation diskutiert und dringend für eine diesbezügliche Ausbildung schon an den Universitäten plädiert. Des Weiteren soll ein ethischer Code formuliert werden, um diesem Behandlungssektor die notwendige Aufmerksamkeit zu vermitteln. Man war sich einig, dass Vorschriften oder Leitlinien hier nicht dienlich sind, sondern nur Konzepte entsprechend den individuellen Möglichkeiten in den entsprechenden Ländern hilfreich sind.

Standespolitisch wurde die Veranstaltung besonders geprägt durch die Teilnahme des FDI-Präsidenten Patrick Hescot, der über aktuelle und künftige Aktivitäten der FDI berichtete. Diese seien erforderlich, da von 135 derzeitigen Mitgliedsländern in der Weltorganisation der Zahnärzte in 100 Ländern ein hoher zahnärztlicher Versorgungsbedarf existiert. Hierzu wird sich im „Thinktank 2020“ die FDI neu positionieren und auf dem World Dental Congress im Herbst in Madrid mit einem Strategieplan neu ausrichten.

Zum Abschluss der Veranstaltung betonte Engel nochmals sehr emotional die Wichtigkeit der internationalen Arbeit, insbesondere vor dem Hintergrund der anhaltenden Diskussionen über die Zukunft der freien Berufe in der Europäischen Union. Hier gelte es, gerade in Gremien wie der ERO und des CED (Council of European Dentists) Stellung zu beziehen, um diesen Tendenzen eine klare Absage zu erteilen. Sonst hätte die freie Berufsausübung ein Ende.

E.-J. Otterbach 